\title{
The process of prediction as a function of the correlation between two scaled variables
}

\author{
CLIFTON W. GRAY, CYNTHIA B. BARNES AND EILEEN F. WILKINSON
}

NORWICH HOSPITAL, NORWICH, CONNECTICUT

\begin{abstract}
College undergraduates were shown pairs of imperfectly correlated values, $\mathrm{X}$ and $\mathrm{Y}$, then shown $\mathrm{X}$ alone and required to predict $Y$. Prediction behavior resembled matching much more than optimizing, and suggests that making predictions on the basis of a single fallible cue is a very difficult task for untrained Ss.
\end{abstract}

\section{Introduetion}

A description of how people perceive two imperfectly correlated variables and use one to predict the other should be useful for at least two reasons. One is that an uncertain environment presumably demands such a process. Another is the fundamental relevance of correlated events to practical problems of decision-making such as those of clinical judgment. With discrete variables, the concept of correlation has been studied both in the simplest ( 2 by 2 ) contingency problem (Smedslund, 1963), and in multiple prediction problems (Estes, 1957). With scaled variables, the concept at the simplest level of two variables has apparently been ignored, although there is a growing body of research on multiple prediction (Summers, 1962; Todd \& Hammond, in press). Work in this neglected region was called for by Smedslund (1963).

\section{Procedure}

The $\mathrm{S}$ was shown sets of paired values, $\mathrm{X}$ and $\mathrm{Y}$, with instructions to learn to make the best estimate of $Y$ from $X$. He was then shown unpaired $X$ values and required to predict $\mathrm{Y}$. $\mathrm{X}$ and $\mathrm{Y}$ were integers one through nine.

In each of two experiments, $\mathrm{S}$ worked three problems in latin-square order, at each of three levels of correlation, called "high," "medium," and "low," $(H, M$, and $L)$, with product-moment $r_{x y}$ of approximately $.96, .75$, and .44 . The values of $\mathrm{X}$ and $\mathrm{Y}$ were presented symbolically (by numerals), physically (by linear distance), or both. In testing, only $\mathrm{X}$ appeared and $\mathrm{S}$ predicted $Y$ in one of two ways: (1) on answer sheets whose format resembled the stimulus display; (2) on a 9 by 9 matrix such that the line of his estimates was visually presented to him while he responded. His guesses are called G. Experiment I varied the length of training trials: each $\mathrm{X}$ was paired with either three, five, or seven $Y$ values, making in all 27, 45, or 63 $X Y$ pairs. Experiment II showed the five $X Y$ pairs per $\mathrm{X}$ that were used in the middle pair-number condition of Experiment I.

Ss were undergraduate women, 72 in Experiment I and 54 in Experiment II.

\section{Results}

Group data. When the Ss were shown an X value, their average prediction was at or near the statistical optimum, $\hat{\mathrm{Y}}$ (Y-hat), but their scatter about these goodlooking averages was extensive. Each $\mathrm{X}$ in testing evoked Gs which spanned a wide range of $Y$, even under high correlation.

For comparison of what the groups predicted in testing and what they had seen in training, all nine Gs from all 72 or all 54 Ss were combined in the same scatter-plots against X. The XY correlations of .96, .75, and .44 in training produced $\mathrm{XG}$ correlations in testing, respectively, of .94, .78, and .53 in Experiment I and $.96, .70$, and .45 in Experiment II. Thus the groups gave Gs that mirrored the scatter-plots of the XY pairs they had seen.

Individual data. Perhaps the most fundamental requirement in this task is to perceive monotonicity, that is, that $\mathrm{Y}$ goes up as $\mathrm{X}$ goes up. While all but a few Ss saw $Y$ as an increasing function of $X$, and 64 of 126 saw it as monotonically increasing under $\mathrm{H}$, only seven did under $M$, and one under $L$.

Table $\mathrm{A}^{1}$ summarizes other aspects of the individual Ss' predictions. The slope, $\mathrm{b}_{\mathrm{gx}}$, of the straight line that best fitted each S's Gs was for the average S correct under high correlation and progressively too high, showing inadequate regression toward the mean, under medium and low. The elevation of that line of best fit, as shown by the mean $G$, was for the average $S$ under all conditions close to the mean, 5, of the actual Ys.

The correlation, $\mathrm{r}_{\mathrm{xg}}$, which shows how much of the variance in S's predictions is accounted for by the $\mathrm{X}$ cue, shows the same result as in the grouped data: $X$ vs. $G$ in the average S's test reflected X vs. $Y$ in the stimuli which he saw in training, except that $r_{x g}$ deviated upward from $r_{x y}$ as $r_{x y}$ decreased.

Perhaps the most interesting summary statistic is $D$, the mean absolute difference across all of an S's Gs between $\mathrm{Y}$-hat, rounded to the nearest integral value, and $G$. D reflects all the other values in Table $A$ and shows S's efficiency as a statistical decision-maker. The hypothesis was tested that $\mathrm{S}$, after seeing an $\mathrm{X}$ during testing, merely fed back a $\mathrm{Y}$ value randomly selected or randomly remembered from among those that had been paired with that $X$. The $D$ values that would be expected under that model were generated by a Monte-Carlo method. When correlation conditions were arranged according to the Ss' accuracy, the order from bad to good was, as expected, low, medium, high. All differences in both experiments were nonchance 
$(p<.01)$. That order changed drastically when correlation conditions were arranged according to how much better Ss did than the model. The model was rejected under low correlation, where the Ss did better $(p<.001$ in Experiment I, $\mathrm{p}<.02$ in Experiment II, two-tailed). Under medium correlation, the Ss also did better, but by a smaller margin $(p<.05$ and $p>.10$ in Experiments I and II). Under high correlation, the model generated better predictions than the Ss in Experiment I $(p<.001)$; they beat the model in Experiment II, but by a chance amount $(p>.05)$. Differences between the Ss and the model, though nonchance, were small. Under all three correlations, several Ss did worse than the worst set of random model Gs.

The $\mathrm{Ss}^{\prime}$ freedom to go beyond the restraints which kept the model close to $\mathrm{Y}$-hat under condition $\mathrm{H}$ does not fully explain why they exceeded the model further under L. The proportion of Gs that fell outside the ranges of Ys that had accompanied each particular X in training was small; in six problems $(H, M$, and $L$ in Experiments I and II), they ranged from .002 to .10. Those proportions are not very closely related (rho $=-.31$ ) to how much better the Ss' Ds were than those of the model. This comparison with the model shows that $\mathrm{Ss}^{\prime}$ behavior was analogous to matching more than to optimizing, since random sampling produces Gs that match Ys. So also does the fact that the XG scatter-plots mirrored the XY scatter-plots. The greater departures from matching toward optimizing occurred under low correlation. That Ss re- sponded to decreased correlation by doing, in this particular sense, a better job of making predictions seems interesting enough to warrant further study.

Table $\mathrm{B}^{1}$ reports the analysis of variance of the experimental variables. Correlation level was significant, $p<.001$, in both experiments. Position effects (improvement across the three problems in each S's latin-square sequence) were significant $(p<.01)$ in Experiment II, but not in Experiment I. Other main effects were attributable to chance in both experiments.

\section{Conclusion}

Although Ss appear to have perceived some covariation between the two variables, and their predictions were clearly better than random guessing, their performance suggests that the task of making predictions on the basis of a single fallible cue is a very difficult one for untrained Ss.

\section{References}

Estes, W. K. Of models and men. Amer. Psychologist, 1957, 10 615-617.

Smedslund, J. The concept of correlation in adults. Scand. J. Psychol., 1963, 4, 165-173.

Summers, S. A. The learning of responses to multiple weighted cues. J. exp. Psychol., 1962, 64, 29-34.

Todd, F. J., \& Hammond, K. R. Differential feedback in two multiple-cue probability learning tasks. Behav. Sci., in press.

\section{Note}

1. A one-page table containing descriptive statistics for individual subjects and a two-page table containing the analyses of variance have been deposited with the office of this journal. Copies may be obtained without charge by writing the journal sending a selfaddressed stamped envelope. 